\title{
System analysis of the quality of meshes in HBIM
}

\author{
Elizaveta Fateeva ${ }^{1, *}$ Vladimir Badenko ${ }^{1}$, Alexandr Fedotov ${ }^{1}$ and Ivan Kochetkov ${ }^{2}$ \\ ${ }^{1}$ Peter the Great St Petersburg Polytechnic University, Civil Engineering Institute, 195251 \\ St Petersburg, Russia \\ ${ }^{2}$ Moscow State University of Civil Engineering, 26 Yaroslavskoye Shosse, 26, Moscow, 129337, \\ Russia
}

\begin{abstract}
Historical Building Information Modelling (HBIM) is nowadays used as a means to collect, store and preserve information about historical buildings and structures. The information is often collected via laser scanning. The resulting point cloud is manipulated and transformed into a polygon mesh, which is a type of model very easy to work with. This paper looks at the problems associated with creating mesh out of point clouds depending on various characteristics in context of façade reconstruction. The study is based on a point cloud recorded via terrestrial laser scanning in downtown Bremen, Germany that contains buildings completed in a number of different architectural styles, allowing to extract multiple architectural features. Analysis of meshes' quality depending on point cloud density was carried out. Conclusions were drawn as to what the rational solutions for effective surface extraction can be for each individual building in question. Recommendations on preprocessing of point clouds were given.
\end{abstract}

\section{Introduction}

Building Information Models (BIMs) are digital representations of buildings and structures that contain information about their life cycle from construction to demolition. Unlike traditional CAD models, where objects only have geometric characteristics, BIMs also represent adjacency relationships between objects as well as other relevant properties such as material characteristics, cost, etc. Creating BIMs significantly reduces resources and time consumed for construction monitoring [1].

BIMs can be applied for both construction in progress and complete construction. BIMs generated in the design stage of a facility are called as-designed BIMs (AD BIMs), whereas BIMs that reflect a facility in its as-built conditions, which this paper is focused on, are called as-built BIMs (AB BIMs).

Creating $\mathrm{AB}$ BIMs is a challenging process for many reasons. While an $\mathrm{AD} B \mathrm{BIM}$ is basically a model to be delivered in real life, an AB BIM requires a lot more work. It involves two major steps: data collection, to capture the as-built conditions, and data modelling, to generate compact, but rich representations [2]. Data collection is often conducted via laser scanning, which produces a 3D point cloud that is later used for data modelling. As of today, there is no uniform method for transforming point clouds into BIMs. Although there is a large

\footnotetext{
* Corresponding author: fateeva.ep@edu.spbstu.ru
} 
number of studies dedicated to this subject, each one of them proposes a different method [39]. The problem here is that every project requires a unique approach that depends on data quality, structural state of a building, software used for both data collection and data modelling, and many other factors. Therefore, the primary objective of studies involving laser scanning is not so much learning techniques and algorithms that allow creating a model, but rather learning to choose an approach that would be the most rational for a specific data set. The end result of data modelling is usually an assembly of surfaces representing façades and/or indoor premises of a structure to which various properties are attached.

Building Information Modelling (BIM) is widely applied for the management of the built heritage. Historical structures are characterized by an enormous complexity in terms of geometry and other factors [10,11], which is why the fast method [12] of collecting geometric data via laser scanning is very effective in this case. Proper data acquisition results in easier search for modelling approaches, which reduces time consumed for data modelling.

In this paper, the authors focuse on approaches suitable for HBIM in the context of façade reconstruction.

\section{Methods}

This study is based on terrestrial laser scanning data recorded in downtown Bremen, Germany (fig. 1). Terrestrial laser scanning allows to scan almost any object, however, there is a number of drawbacks such as expensive equipment and availability of educational resources and/or a qualified expert in laser scanning, considerable weight of the equipment itself and, thus, inconvenience of its transportation, frequent measurement inaccuracies, especially with highly reflective surfaces and dark objects. Furthermore, processing of the collected 3D point clouds is highly time-consuming and costly due to software limitations. However, despite the drawbacks, this approach is still considered to be the most accurate.

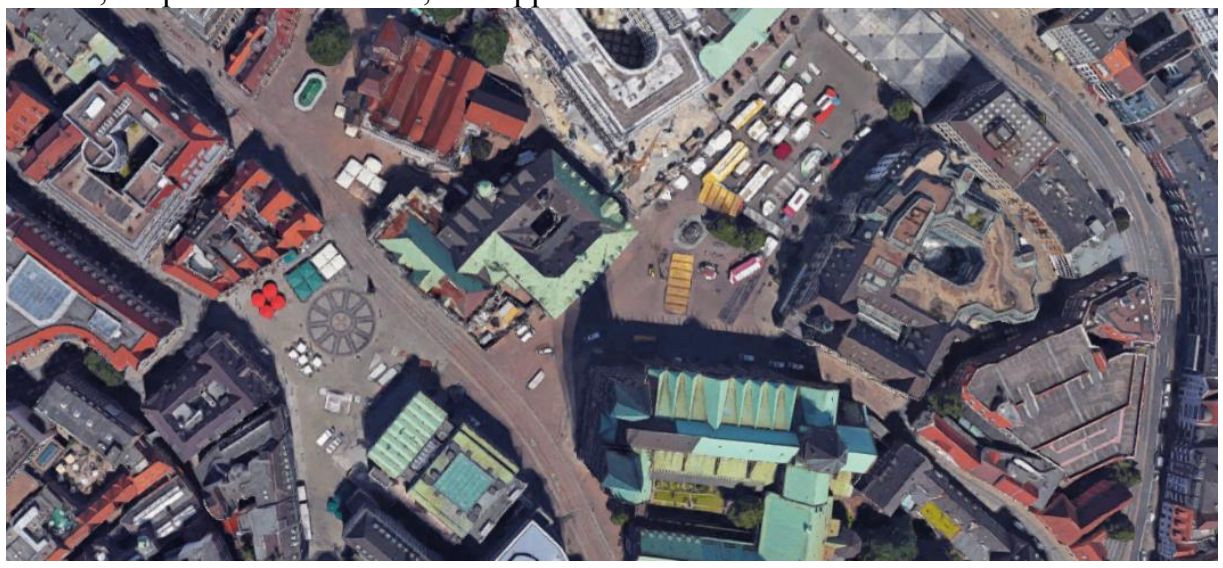

Fig. 1. Aerial view of the scanned area.

Using historic data to restore or conserve historic and buildings is common in the wider area of conservation and is a wide area of research. The use of architectural knowledge to inform the creation of models is becoming a common part of a design approach within research areas of architectural heritage modelling [13-17]. Most works in this area differ in their analysis of historic architectural data. HBIM focuses on the emergence of architectural pattern books to define architectural rules and detail, taking into account the evolution and form of classical architecture [18]. 
This study is focused on correspondence between surface density of point clouds, materials of which buildings were constructed and the quality of the resulting mesh in comparison with the actual facade.

\section{Results}

In this study, a series of comparisons between triangle meshes and the actual façades was conducted. Façades in each comparisons had a different amount of highly reflective surfaces and architectural features. The density of each point cloud containing the façade was calculated in the same neighborhood radius.

1. A historical building with very little amount of highly reflective surfaces (i.e. windows) (fig. 2). It can be seen that the resulting mesh (fig 3) has a very high detalization due to the initial high point cloud density. However, since there is not many complex features on the façade, the point cloud density is excessive in some of its areas and may be reduced. This will allow easier manipulation of the point cloud.

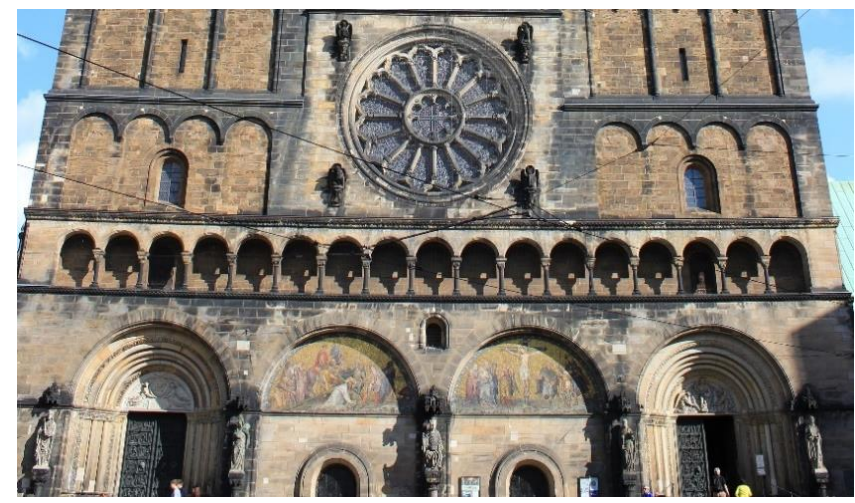

Fig. 2. The façade of the building with the least amount of highly reflective surfaces.

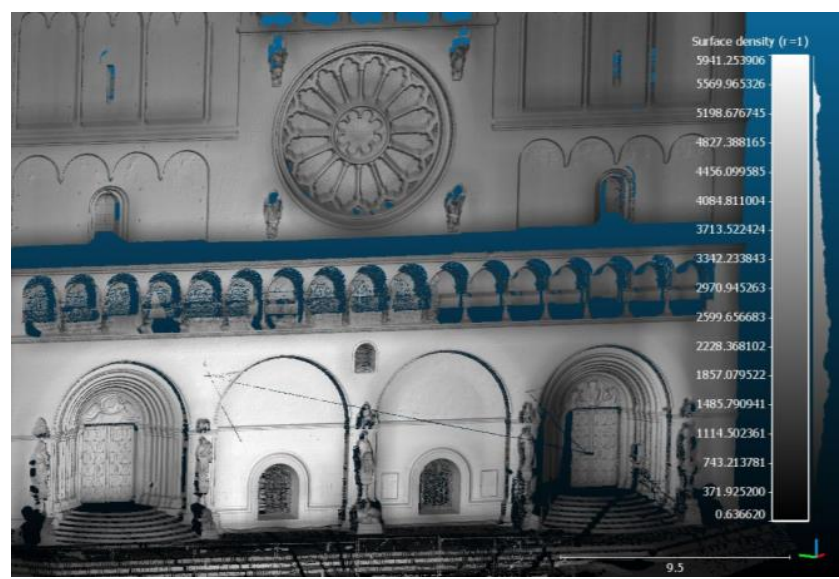

Fig. 3. The resulting mesh of the building with the least amount of highly reflective surfaces.

2. A historical building with a medium amount of highly reflective surfaces (fig. 4). The initial point cloud of the façade has a lower density. However, for simple features it still is excessive. The mesh's detalization remains very high (fig. 5). 
Obviously, the quality of the mesh heavily depends on the complexity of surface. The average density of point clouds forming sculptures on fig. 6 and 7 differs by about 1,5 times. The detalization of the mesh on fig. 6 is much higher.

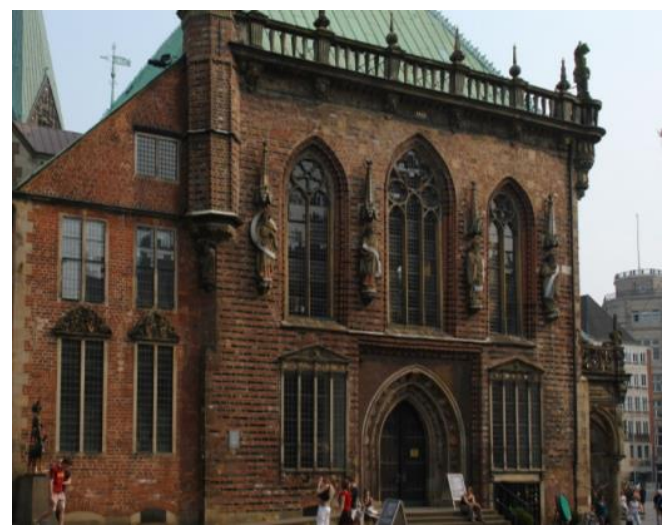

Fig. 4. The façade of the building with a medium amount of highly reflective surfaces.

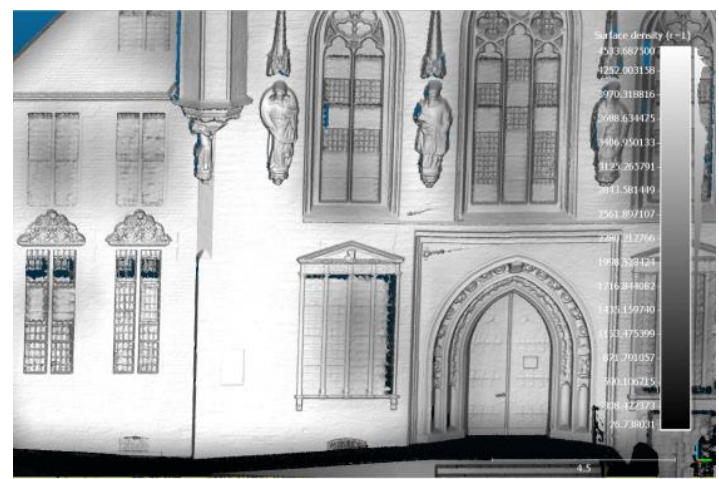

Fig. 5. The resulting mesh of the building with a medium amount of highly reflective surfaces.

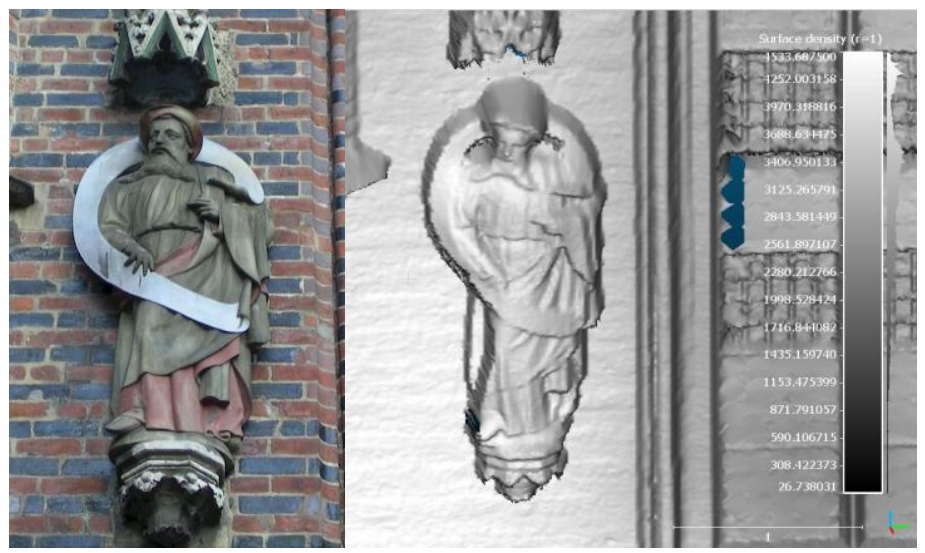

Fig. 6. A feature on the façade (left) and the constructed mesh (right). 


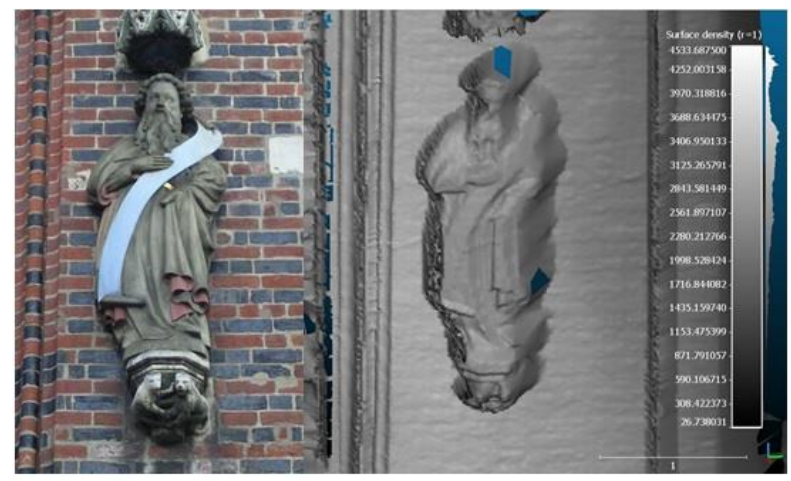

Fig. 7. A feature on the façade (left) and the constructed mesh (right).

Architectural features like that are often a part of the ensemble of historical buildings' façades, therefore, it is important to understand the complexity and operation order needed to achieve a high-quality surface for HBIM purposes.

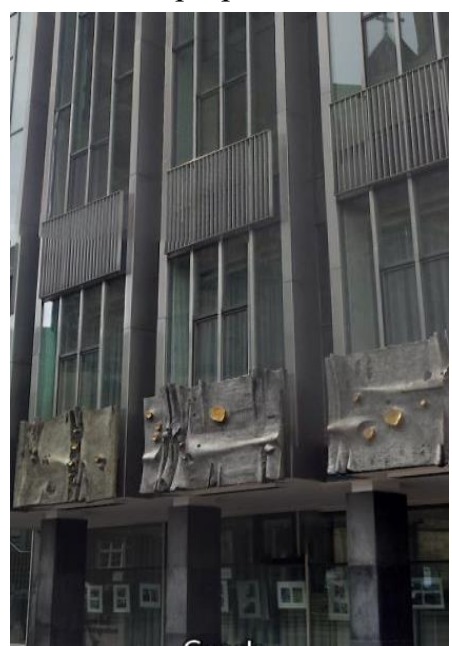

Fig. 8. The façade of the building with a large amount of highly reflective surfaces.

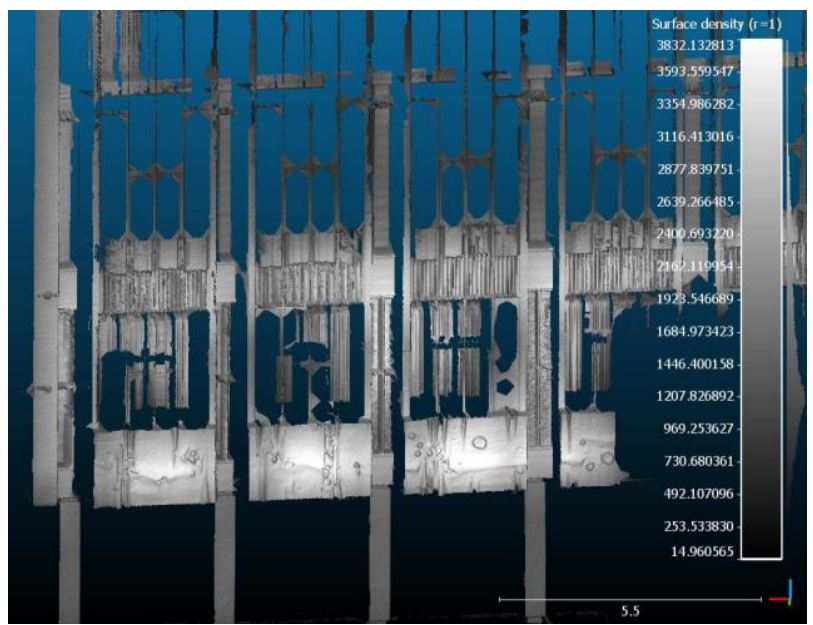

Fig. 9. The resulting mesh of the building with a large amount of highly reflective surfaces. 
3. A building with a large amount of highly reflective surfaces (fig. 8). This is where most problems with façade reconstruction occur. The abundance of windows on the façade results in an inconsistent point cloud with many gaps even with overall high density (fig. 9). Constructing a high-quality mesh from it can be a challenge, since the software usually does not leave the gaps as they are but closes them in a random order.

\section{Discussion}

The higher detalization of the resulting mesh compared to the real appearance of the façade can be achieved with a higher point cloud density. For this purpose, a set of point clouds recorded from several positions is preferred, since this will allow a wider overview of the façade's features and, therefore, the resulting quality of the constructed mesh will be closer to the real-life façade appearance [19-22].

The biggest challenge in façade reconstruction by far is the highly reflective surfaces. Obviously, most buildings contain a lot of windows which are represented as irregular gaps in the point cloud. In order to solve this problem, an algorithm is needed that will allow to either make the gaps regular or to achieve their smooth closing.

For the easier model handling meshes can be simplified (i.e. the number of triangles can be reduced) where it is appropriate. For instance, some planar surfaces like walls usually have a very high point cloud density due to the lack of curvatures and contain abundant information about their roughness. The degree of simplification depends on the purpose of the final model.

\section{Conclusions}

A point cloud recorded in downtown Bremen, Germany was analyzed. A set of façades with different architectural features and amount of highly reflective surfaces was evaluated. Conclusions were drawn as to how to extract a better-quality mesh depending on the façade's features. Recommendations on additional point cloud and mesh transformation were given.

\section{Acknowledgements}

The research was supported by Ministry of Education and Science of Russia within the framework of the Federal Program "Research and Development in Priority Areas for the Development of the Russian the Science and Technology Complex for 2014-2020" (project ID RFMEFI58417X0025).

\section{References}

1. P. Tang, D. Huber, B. Akinci, R. Lipman, A. Lytle, Automat. Constr., 19, 829-843 (2010)

2. V. Pătrăucean, I. Armeni, M. Nahangi, J. Yeung, I. Brilakis, C. Haas, Adv. Eng. Inform., 29.2, 162-171 (2015).

3. L. Inzerillo, C. Santagati, M. Galizia, XIII International Forum Le Vie dej Mercanti, (2015).

4. J. Heo, S. Jeong, H.-K. Park, J. Jung, S. Han, S. Hong, H.-G. Sohn, Comput Environ Urban Syst, 41, 26-38 (2013). 
5. B. Yang, Z. Dong, ISPRS J Photogramm Remote Sens, 81, 19-30 (2013).

6. S. Barnea, S. Filin, ISPRS J Photogramm Remote Sens, 76, 33-48 (2013).

7. B.S. Yang, Z. Wei, Q. Li, J. Li, IEEE Trans. Geosci. Remote Sens, 10 (4), 766-770 (2013).

8. B.S. Yang, Z. Wei, Q. Li, J. Li, Int J Remote Sens, 33 (18), 5839-5861, 2012.

9. Ch. Wang, Y. K. Cho, Ch. Kim, Automat. Constr., 56, 1-13 (2015).

10. H. Son, F. Bosché, C. Kim, Adv. Eng. Inform., 29(2), 172-183 (2015).

11. L. Barazzetti, Adv. Eng. Inform., 30(3), 298-311 (2016).

12. V. Badenko, D. Zotov, A. Fedotov, E3S Web of Conferences, 33, 1074 (2018).

13. C. Chevrier, N. Charbonneau, P. Grussenmeyer, J. Perrin, IJAC, 8 (2), 131-146 (2010).

14. C. Chevrier, Y. Maillard, J.P. Perrin, ISPRS Archives, 38 (2009)

15. L. De Luca, C. Busayarat, C. Stefani, P. Véron, M. Florenzano, Comput Graph 35 (2), 227-241 (2011).

16. P. Muller, P. Wonka, S. Haegler, A. Ulmer, L. Van Gool, ACM Trans Graph, 25 (3), 614-623 (2006).

17. P. Wonka, M. Wimmer, F. Sillion, W. Ribarsky, ACM Trans Graph, 22 (3), 667-669 (2003).

18. M. Murphy, E. McGovern, S. Pavia, ISPRS J Photogramm Remote Sens, 76, 89-102 (2013).

19. R. Hartley, A. Zisserman, Multiple View Geometry in Computer Vision, CUP (2003).

20. D.G. Lowe, Int. J. Comput. Vis., 60 (2), 91-110 (2004).

21. T. Tuytelaars, K. Mikolajczyk, Comput. Graph. Vis., 3 (3), 177-280 (2008).

22. P. Musialski, P. Wonka, D.G. Aliaga, M. Wimmer, L. van Gool, W. Purgathofer, Comput. Graph. Forum, 32 (6), 146-177, (2013). 